\title{
Brain-Derived Neurotrophic Factor and Its Associations with Metabolism and Physical Activity in a Latino Sample
}

\author{
Rodrigo Fonseca-Portilla, ${ }^{1, *}$ Janina Krell-Roesch, PhD, ${ }^{2}$ Gabriel Q. Shaibi, PhD, ${ }^{3}$ Richard J. Caselli, MD, ${ }^{4}$ \\ Lawrence J. Mandarino, PhD, ${ }^{5}$ Nan Zhang, MS, ${ }^{6}$ Joseph G. Hentz, MS, ${ }^{6}$ Dawn K. Coletta, PhD, \\ Elena Anna de Filippis, MD, PhD, ${ }^{7}$ Sara Dawit, MD, ${ }^{4}$ and Yonas E. Geda, MD, MSc ${ }^{2,4,8}$
}

\begin{abstract}
Background: Brain-derived neurotrophic factor (BDNF) is associated with body weight and other health conditions but remains understudied in the Latino population. The aim of this study was to examine the associations of BDNF serum levels with body mass index (BMI), physical activity, and the rs6265 nonconservative polymorphism among 349 Latinos aged $\geq 18$ years enrolled in the Arizona Insulin Resistance Registry.

Materials and Methods: Data on physical activity were acquired using a self-reported questionnaire. BDNF serum levels were measured utilizing a modified ELISA method, and the rs6265 polymorphism was genotyped by the Assay-by-Design service. Two sample $t$-tests or chi-squared tests were employed to compare demographics and outcomes between physically active and nonactive groups as well as between rs6265 CC and CT+TT groups.

Results: BDNF levels and rs6265 polymorphism did not differ significantly between the physically active $(N=195)$ and nonactive group $(N=154)$. Participants with the rs6265 polymorphism did not show any significant difference in BDNF levels or BMI when compared with those with the normal functional variant. Higher BDNF levels were significantly associated with higher age $(r=0.11, P=0.04)$ and higher 2-hr glucose level $(r=0.11, P=0.04)$.

Conclusions: In this cross-sectional study, the rs6265 polymorphism was not associated with a higher risk of obesity, or lower circulating levels of BDNF. Thus, the rs6265 polymorphism may have a different impact in Latinos as compared with other previously studied populations.
\end{abstract}

Keywords: BDNF, metabolism, physical activity, Latinos

\section{Introduction}

$\mathrm{B}$ RAIN-DERIVED NEUROTROPHIC FACTOR (BDNF) plays an important role in the development, regulation, and survival of neurons in the hippocampus, and other structures relevant to cognitive function. ${ }^{1}$ BDNF deficiency has been associated with neurocognitive disorders and metabolic diseases. ${ }^{2-5}$ Furthermore, BDNF is also associated with physical activity. ${ }^{6-11}$ Although the exact mechanism of action remains unknown, it has been hypothesized that BDNF could be one factor accounting for the beneficial effects of physical activity on neurocognitive function, by promoting

\footnotetext{
${ }^{1}$ Anáhuac University School of Medicine, Mexico City, Mexico.

${ }^{2}$ Mayo Clinic Translational Neuroscience and Aging Program, Mayo Clinic, Scottsdale, Arizona.

${ }^{3}$ Center for Health Promotion and Disease Prevention, Arizona State University, Phoenix, Arizona.

${ }^{4}$ Department of Neurology, Mayo Clinic, Scottsdale, Arizona.

${ }^{5}$ Department of Medicine, University of Arizona College of Medicine, Tucson, Arizona.

Departments of ${ }^{6}$ Biostatistics, ${ }^{7}$ Endocrinology, and ${ }^{8}$ Psychiatry and Psychology, Mayo Clinic, Scottsdale, Arizona.

*R. Fonseca-Portilla is a medical student who did research training under the mentorship of Professor Geda at Mayo Clinic in Scottsdale, Arizona.
}

(C) Rodrigo Fonseca-Portilla et al. 2018; Published by Mary Ann Liebert, Inc. This Open Access article is distributed under the terms of the Creative Commons Attribution Noncommercial License (http://creativecommons.org/licenses/by-nc/4.0/) which permits any noncommercial use, distribution, and reproduction in any medium, provided the original author(s) and the source are cited. 
cell growth and survival in the hippocampal neurons. ${ }^{6}$ In addition, interaction effects between genetics with physical activity and metabolic parameters have been reported. ${ }^{7}$ BDNF is implicated in the regulation of food intake and body weight, by suppressing food intake through hippocampal signaling and enhancing energy expenditure. ${ }^{5,8,9}$ Although the pathology of obesity is multifactorial, studies have reported an inverse association between lower BDNF serum concentration and higher body mass index (BMI) and percentage of body fat. ${ }^{10-12}$

The BDNF gene is located on chromosome 11p14.1. Considerable allele and haplotype diversity in the BDNF gene has been observed among different ethnic populations. It has been reported that there is a specific genetic variant within the gene that may influence the risk of obesity, Alzheimer's disease, cognitive impairment, and other health conditions. ${ }^{8,13-17}$ There is a well-identified single nucleotide polymorphism (SNP) expressed in the pro-region of the BDNF gene on position 66 (change from valine to methionine): rs6265. ${ }^{14}$ This change results in aberrant sorting of BDNF into secretory vesicles and decreased activitydependent secretion. ${ }^{14}$ The rs6265 variant is of particular interest, because it is the only known frequent nonconservative polymorphism in the BDNF gene ${ }^{12}$ and is associated with various health conditions, such as obesity and type 2 diabetes. $^{12,15,18}$

To date, the majority of BDNF studies have been conducted in animal models. $5,9,19,20$ The few published human studies predominantly involve Caucasian or Asian populations and reported conflicting results on the associations between health conditions, particularly obesity, and the rs6265 polymorphism. ${ }^{10,12,15,18}$ The association of the rs6265 SNP and BMI has not been described among Latinos who are the fastest growing minority group in the United States. ${ }^{21}$ Latinos are disproportionately affected by obesity and have a high prevalence of chronic diseases such as diabetes and cardiovascular disease. ${ }^{22}$ According to the National Hispanic Council on Aging, Latinos engage in less physical activity than non-Hispanic whites, which may put them at higher risk for metabolic disorders. ${ }^{23,24}$ Therefore, the aim of our study was to investigate the cross-sectional associations between the BDNF rs6265 polymorphism, physical activity, and BMI among a community-based sample of Latinos residing in Phoenix, Arizona. We hypothesized that higher serum BDNF levels would be associated with a higher level of physical activity and lower BMI. In addition, we hypothesized that there would be a higher frequency of the BDNF rs6265 mutation among participants who had lower serum BDNF levels and reported less physical activity.

\section{Materials and Methods}

\section{Study participants}

This study utilized existing stored biospecimens and previously collected data from participants enrolled in the Arizona Insulin Resistance Registry. In brief, individuals who identified themselves as Latino or Mexican American between the ages of 18 and 85 years were recruited through community clinics, flyers, and word of mouth. ${ }^{25}$ Participants completed a detailed medical history and an intake questionnaire. Engagement in physical activity was assessed through one item from the intake questionnaire: "Do you participate in exercise? Yes/No." If participants answered "yes," then further details were obtained regarding intensity, frequency, and duration. The physical examination included anthropometric measures (height, weight, and waist circumference), seated blood pressure, fasting laboratory measures (comprehensive metabolic and lipid panel), and an oral glucose tolerance test. In addition, participants provided consent to collect and bank fasting serum, DNA, and RNA for future use. All data were entered into a searchable REDCap database and biospecimens were stored at $-80^{\circ} \mathrm{C}$ in individual aliquots at Arizona State University (ASU) laboratories, housed in the Mayo Clinic Arizona campus. The study was approved by the Institutional Review Board of Arizona State University. Written informed consent was obtained from each participant before participation.

\section{Laboratory procedure}

We used Mayo Clinic's Biospecimens Accessioning and Processing laboratory to process the BDNF assays. BDNF levels were measured in the serum, given that serum BDNF levels are reflective of brain BDNF. ${ }^{26}$ To analyze serum BDNF levels, we used a modified ELISA method. ${ }^{27}$ SNP genotyping for rs6265 was performed by the Assay-byDesign service (Applied Biosystems, CA.), as previously described. $^{28}$ In brief, in a 384 -well plate, $2 \mu \mathrm{L}$ of purified genomic DNA $(2 \mathrm{ng} / \mu \mathrm{L})$ were incubated with primers and probes with the SNP of interest $(0.09 \mu \mathrm{L}), 3.5 \mu \mathrm{L}$ of TaqMan Universal Polymerase Chain Reaction Master Mix-No AmpErase UNG, and $1.14 \mu \mathrm{L}$ of distilled water. Samples were polymerase-chain-reaction-amplified on the Applied Biosystems 9700HT Thermal Cycler under the following conditions: denatured for $10 \mathrm{~min}$ at $95^{\circ} \mathrm{C}$, denatured, annealed, and extended for 40 cycles of $15 \mathrm{~s}$ at $92^{\circ} \mathrm{C}$ and $1 \mathrm{~min}$ at $60^{\circ} \mathrm{C}$. The $384-w e l l$ microplates were scanned for fluorescence emission using a $7900 \mathrm{HT}$ sequence detector (Applied Biosystems), and alleles were scored using the allelic discrimination software Sequence Detection System v2.3 (Applied Biosystems).

\section{Statistical analysis}

Age, BMI, fasting glucose, 2-hr glucose, and serum BDNF levels of participants who reported physical activity versus those who did not report physical activity were compared using two-sample $t$-test. Gender and rs6265 SNP groups (i.e., CC, CT, and TT) were compared between physically active and nonactive participants using chi-squared test. Additional statistical comparisons between groups (i.e., between CC and CT+TT combined rs6265 genotype groups) were made by using the twosample $t$-test or chi-squared test. The associations between BDNF and age, BMI, and glucose (fasting or 2-hr) were quantified by using the Pearson correlation coefficient. With 225 patients in the $\mathrm{CC}$ group, and 118 patients in the CT+TT group, we have $80 \%$ power to detect an effect size 0.32 or larger (small to medium effect size) in a continuous phenotype trait between the two groups, assuming a 0.05 twosided significance level. Statistical testing was performed at the conventional two-tailed $\alpha=0.05$ using SAS software Version 9.3 (SAS Institute, Inc.). 
Table 1. Demographics, Body Mass Index, Glucose, and Brain-Derived Neurotrophic Factor Levels; Stratified by Physical Activity Group

\begin{tabular}{|c|c|c|c|c|c|}
\hline & \multicolumn{2}{|c|}{ Active } & \multirow[b]{2}{*}{$\Delta$} & \multirow[b]{2}{*}{$95 \% C I$} & \multirow[b]{2}{*}{$\mathrm{P}$} \\
\hline & $\operatorname{Yes}(\mathrm{N}=195)$ & No $(\mathrm{N}=154)$ & & & \\
\hline Women & $118(61 \%)$ & $115(75 \%)$ & $-14.2 \%$ & -23.9 to -4.5 & 0.005 \\
\hline Age (years); mean (SD) & $41.7(8.3)$ & $41.5(9.4)$ & $0.2(8.8)$ & -1.7 to 2.1 & 0.82 \\
\hline BMI $\left(\mathrm{kg} / \mathrm{m}^{2}\right) ;$ mean (SD) & $29.5(4.9)$ & $31.9(7.5)$ & $-2.3(6.2)$ & -3.6 to -1.0 & $<0.001$ \\
\hline \multicolumn{6}{|l|}{ Glucose $(\mathrm{mg} / \mathrm{dL})$} \\
\hline Fasting; mean (SD) & $96(31)$ & $99(40)$ & $-3(34)$ & -10 to 4 & 0.41 \\
\hline $2-\mathrm{Hr} ;$ mean $(\mathrm{SD})$ & $140(61)$ [ $N$ 189] & $150(70)\left[\begin{array}{ll}N & 150\end{array}\right]$ & $-11(65)$ & -25 to 3 & 0.13 \\
\hline \multicolumn{6}{|l|}{ rs6265 } \\
\hline $\mathrm{N}$ & 193 & 150 & & & 0.46 \\
\hline $\mathrm{CC}$ & $123(64 \%)$ & $102(68 \%)$ & & & \\
\hline CT & $66(34 \%)$ & $43(29 \%)$ & & & \\
\hline TT & $4(2 \%)$ & $5(3 \%)$ & & & \\
\hline BDNF $(\mathrm{ng} / \mathrm{mL})$; mean $(\mathrm{SD})$ & $33(16)$ & 34 (17) & $-1(16)$ & -4 to 3 & 0.64 \\
\hline
\end{tabular}

$\Delta$, difference in means between active and nonactive groups; BDNF, brain-derived neurotrophic factor serum level; BMI, body mass index; 95\% CI, 95\% confidence interval; CC, CC homozygotes; CT, CT heterozygotes; rs6265, rs6265 single nucleotide polymorphism; $\mathrm{SD}$, standard deviation; TT, TT homozygotes.

\section{Results}

Data were available on 349 participants (233 women and 116 men) with a mean age of 42 years. Mean $( \pm \mathrm{SD}) \mathrm{BDNF}$ levels did not differ $(P=0.89)$ between men $(33 \pm 16 \mathrm{ng} / \mathrm{mL})$ and women $(33 \pm 17 \mathrm{ng} / \mathrm{mL})$. There were 195 participants $(56 \%)$ who reported engaging in regular physical activity (i.e., "active group"). The active group had a significantly smaller proportion of women, and a lower mean BMI than the nonactive group. Mean BDNF levels did not differ significantly between the active and nonactive group $(\Delta=-1 \mathrm{ng} / \mathrm{mL}, 95 \% \mathrm{CI}-4$ to $3, P=0.64)$. Information on rs6265 genotype was available for 343 participants. Two hundred twenty-five participants $(64 \%)$ were CC homozygous for the functional variant, $109(31 \%)$ were CT heterozygous, and $9(3 \%)$ were TT homozygous for the mutation. The minor allele frequency in our population was 0.1851 . There was no significant difference in rs6265 genotype frequency between the physically active and nonactive group. Adjusting for age, gender, BMI, fasting glucose, or 2-hr glucose did not alter the results (Table 1).

Mean BDNF levels did not differ between the CC and CT genotypes. Similarly, there were no differences regarding any other demographic characteristics between $\mathrm{CC}$ and CT genotypes (Table 2). Higher BDNF levels were significantly associated with higher age $(r=0.11, P=0.04)$ and higher 2-hr glucose level ( $r=0.11, P=0.04$; Fig. 1$)$. In addition, there was a marginally significant association between higher BDNF levels and higher fasting glucose levels $(r=0.10$, $P=0.07)$. BDNF level was not associated with BMI $(r=-0.05$, $P=0.36)$.

\section{Discussion}

Building upon the growing body of research on BDNF and its implication among different populations, we sought to determine the cross-sectional association between BDNF serum levels with BMI, physical activity, and the rs6265 polymorphism in a convenience sample of Latinos residing in Phoenix, Arizona. We observed that physical activity and the rs6265 polymorphism did not have any significant association with BDNF serum concentrations. In addition, the rs6265 polymorphism was also not associated with BMI in our sample.

To date, we have not identified a study that examined the association between rs6265 BDNF polymorphism and physical activity or BMI among Latinos. Given that physical activity is associated with increased BDNF expression,

Table 2. Demographics, Body Mass Index, Glucose, and Brain-Derived Neurotrophic Factor Levels; Stratified by CC AND CT+TT RS6265 Genotype Group

\begin{tabular}{lccccc}
\hline & \multicolumn{1}{c}{$\begin{array}{c}C C \\
(\mathrm{~N}=225)\end{array}$} & $\begin{array}{c}C T+T T \\
(\mathrm{~N}=118)\end{array}$ & $\Delta$ & $95 \% C I$ & $\mathrm{P}$ \\
\hline Women & $151(67.1 \%)$ & $77(65.3 \%)$ & $1.9 \%$ & -8.7 to 12.4 & 0.73 \\
Age (years); mean (SD) & $41.3(8.5)$ & $42.4(9.4)$ & $-1.1(8.8)$ & -3.1 to 0.9 & 0.26 \\
BMI (kg/m $) ;$ mean (SD) & $30.5(6.5)$ & $30.7(5.7)$ & $-0.2(6.2)$ & -1.6 to 1.2 & 0.80 \\
Obese (BMI $\geq 30)$ & $101(44.9 \%)$ & $57(48.3 \%)$ & $-3.4 \%$ & -14.5 to 7.7 & 0.55 \\
Glucose (mg/dL) & & & & & \\
$\quad$ Fasting; mean (SD) & $99(35)$ & $96(33)$ & $3(34)$ & -5 to 11 & 0.46 \\
2-Hr; mean (SD) & $146(70)[N 220]$ & $141(56)[N 113]$ & $5(66)$ & -9 to 19 & 0.46 \\
Active & $123(54.7 \%)$ & $70(59.3 \%)$ & $-4.7 \%$ & -15.6 to 6.3 & 0.41 \\
BDNF (ng/mL); mean (SD) & $34(17)$ & $32(15)$ & $1(16)$ & -2 to 5 & 0.49 \\
\hline
\end{tabular}

$\Delta$, difference in means between CC homozygotes and CT heterozygotes. 
FIG. 1. Correlation between BDNF serum level (in $\mathrm{ng} / \mathrm{mL}$ ) and 2-hr glucose level (in $\mathrm{mg} / \mathrm{dL}$ ). BDNF, brain-derived neurotrophic factor.

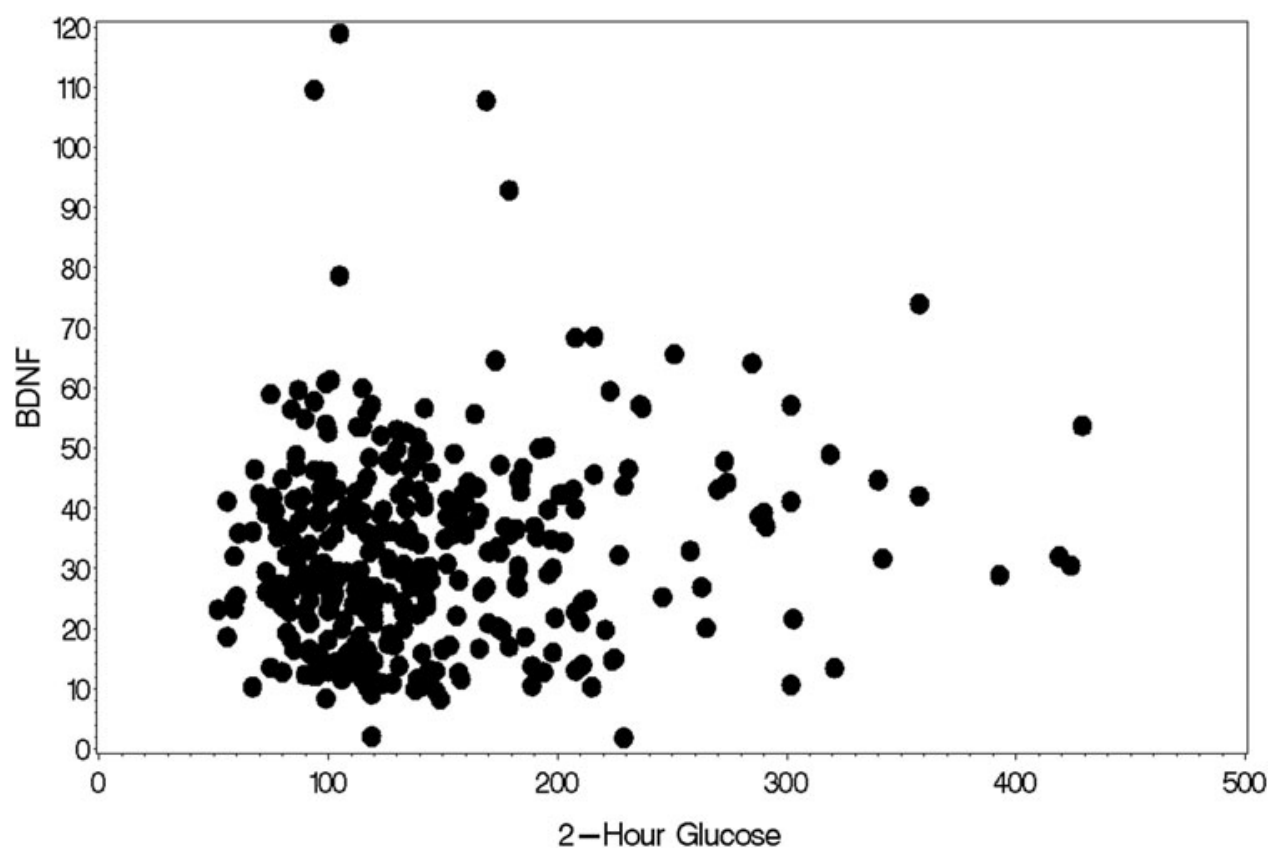

primarily in the hippocampus, ${ }^{19,29,30}$ we hypothesized that a higher level of physical activity would be associated with higher BDNF concentrations. However, we did not observe a significant difference in BDNF levels between the physically active and nonactive group. This may in part be explained by the relatively high BMI of the participants (including those who reported physical activity), which was mostly at overweight or obese levels. This is relevant since BMI has been reported to be directly affected by secreted levels of BDNF, undermining any potential effect that physical activity could have on BDNF expression. ${ }^{5}$ Another possible explanation is that BDNF response is transient after acute exercise and does not have a lasting effect. ${ }^{31,32}$ In our study we did not measure BDNF after an acute bout of exercise, rather we used a survey to acquire data on regular physical activity. ${ }^{33}$ This may explain the absence of difference in BDNF levels between the physically active and nonactive group in our study.

We also investigated the association between the rs6265 SNP and serum BDNF level among Latinos. The frequency and distribution of methionine carriers (CT and TT) in our sample is consistent with previous studies that report BDNF genotype distributions in primarily non-Hispanic white samples. ${ }^{34}$ In our study, mean BDNF levels did not vary between CC and CT genotypes. Similar studies conducted in Chinese and Indian ${ }^{35,36}$ populations reported that BDNF levels are not affected by the rs6265 polymorphism, supporting our findings that BDNF levels may not vary by genotype status.

Furthermore, we did not observe an association between the rs6265 polymorphism and BMI in our study sample. There are conflicting results as to whether rs6265 genotype is associated with BMI across populations. ${ }^{12,13,15,18}$ For example, investigators from Mexico found that the rs6265 polymorphism confers a lower risk for obesity. ${ }^{16}$ However, a study in a Boston Puerto Rican sample reported a genderspecific association, that is, $\mathrm{CC}$ men had twice the risk of being overweight, whereas $\mathrm{CC}$ women had a lower mean
BMI. ${ }^{8}$ Whereas we did not observe any gender- or agespecific associations, which could in part be explained by the fact that our population was on average 10 years younger than the Puerto Rican sample.

Finally, we observed weak correlations between BDNF levels with glucose levels and age. BDNF has been reported to impact metabolism of peripheral organs, ${ }^{2}$ and as a result, BDNF may be elevated after glucose ingestion, possibly playing a role in body weight balance. ${ }^{11}$ As for the association with age, further research is needed to determine the cause of the relationship with BDNF, since independent agerelated increase has been reported in only one study. ${ }^{37}$

There are other possible explanations for the discrepancies between this study's findings and previous reports. For example, lifestyle factors such as low physical activity and poor diet may contribute to high obesity rates among Latinos and may also interact with genetic effects. In addition, poor socioeconomic status and long working hours may make it more difficult to adhere to healthy lifestyles and diet. Furthermore, lack of education may be a risk factor for metabolic syndrome and other chronic diseases. Finally, genetic variability may be another explanation. As mentioned earlier, most studies have used European and Caucasian samples and distribution of genotypes and phenotypes may differ between populations.

Our findings should be interpreted within the context of strengths and limitations of this study. The strengths of our study include a relatively large, community-based, low socioeconomic sample of Latino participants residing in Arizona. The main limitation of this study is a lack of genetic markers that would have enabled us to provide information on the ancestry groups or genetic substructure of our nonhomogenous sample. Furthermore, we were not able to rule out potential bias that may stem from genetic admixture or population stratification. However, potential bias due to these factors may be of less relevance to our study since we are not reporting a positive finding. The cross-sectional design is one of the limitations of our study. Such a design 
does not indicate the direction of causality. In addition, physical activity was assessed using a survey that may be susceptible to recall bias.

In summary, in this cross-sectional study among Latinos residing in Phoenix, Arizona, serum BDNF levels were not significantly associated with physical activity, BMI, or the rs6265 polymorphism. The rs6265 polymorphism was not associated with a higher risk of obesity or lower circulating levels of BDNF. Thus, the rs6265 polymorphism may have a different impact in Latinos as compared with other previously studied populations. Future prospective studies in a larger sample of Latinos are needed to confirm this preliminary finding.

\section{Acknowledgments}

The study was supported by the Mayo Clinic Center for Individualized Medicine. It was in part supported by the Robert Wood Johnson Foundation, the Research Education Component of Arizona Alzheimer's Disease Core Center (P30AG19610), and the Arizona Alzheimer's Research Consortium. The authors thank Eric A. Miller who contributed to this work when he was doing research training under the mentorship of Professor Geda at Mayo Clinic in Scottsdale, Arizona.

\section{Author Disclosure Statement}

No conflicting financial interests exist.

\section{References}

1. Huang EJ, Reichardt LF. Neurotrophins: Roles in neuronal development and function. Annu Rev Neurosci 2001;24: 677-736.

2. Singh RT, Tokunaga M, Wilczynska A, et al. Effect of brain derived neurotrophic factor, in relation to diet and lifestyle factors, for prevention of neuropsychiatric and vascular diseases and diabetes. Open Nutr J 2014;7:5-14.

3. Phillips HS, Hains JM, Armanini M, et al. BDNF mRNA is decreased in the hippocampus of individuals with Alzheimer's disease. Neuron 1991;7:695-702.

4. Howells DW, Porritt MJ, Wong JY, et al. Reduced BDNF mRNA expression in the Parkinson's disease substantia nigra. Exp Neurol 2000;166:127-135.

5. Nakagawa T, Tsuchida A, Itakura $\mathrm{Y}$, et al. Brain-derived neurotrophic factor regulates glucose metabolism by modulating energy balance in diabetic mice. Diabetes 2000;49: 436-444.

6. Carro E, Trejo JL, Busiguina S, et al. Circulating insulinlike growth factor I mediates the protective effects of physical exercise against brain insults of different etiology and anatomy. J Neurosci 2001;21:5678-5684.

7. Kim JY, DeMenna JT, Puppala S, et al. Physical activity and FTO genotype by physical activity interactive influences on obesity. BMC Genet 2016;17:47.

8. Ma XY, Qiu WQ, Smith CE, et al. Association between BDNF rs6265 and obesity in the Boston Puerto Rican Health Study. J Obes 2012;2012:102942.

9. Kernie SG, Liebl DJ, Parada LF. BDNF regulates eating behavior and locomotor activity in mice. EMBO J 2000;19: 1290-1300.

10. Suwa M, Kishimoto H, Nofuji Y, et al. Serum brain-derived neurotrophic factor level is increased and associated with obesity in newly diagnosed female patients with type 2 diabetes mellitus. Metabolism 2006;55:852-857.

11. Lee IT, Wang JS, Fu CP, et al. Relationship between body weight and the increment in serum brain-derived neurotrophic factor after oral glucose challenge in men with obesity and metabolic syndrome: A prospective study. Medicine 2016;95:e5260.

12. Friedel S, Horro FF, Wermter AK, et al. Mutation screen of the brain derived neurotrophic factor gene (BDNF): Identification of several genetic variants and association studies in patients with obesity, eating disorders, and attentiondeficit/hyperactivity disorder. Am J Med Genet B Neuropsychiatr Genet 2005;132B:96-99.

13. Wu L, Xi B, Zhang M, et al. Associations of six single nucleotide polymorphisms in obesity-related genes with BMI and risk of obesity in Chinese children. Diabetes 2010;59:3085-3089.

14. Egan MF, Kojima M, Callicott JH, et al. The BDNF val66met polymorphism affects activity-dependent secretion of BDNF and human memory and hippocampal function. Cell 2003;112:257-269.

15. Beckers S, Peeters A, Zegers D, et al. Association of the BDNF Val66Met variation with obesity in women. Mol Genet Metab 2008;95:110-112.

16. Morales-Marin ME, Genis-Mendoza AD, Tovilla-Zarate CA, et al. Association between obesity and the brainderived neurotrophic factor gene polymorphism Val66Met in individuals with bipolar disorder in Mexican population. Neuropsychiatr Dis Treat 2016;12:1843-1848.

17. Ribeiro L, Busnello JV, Cantor RM, et al. The brainderived neurotrophic factor rs6265 (Val66Met) polymorphism and depression in Mexican-Americans. Neuroreport 2007;18:1291-1293.

18. Gunstad J, Schofield P, Paul RH, et al. BDNF Val66Met polymorphism is associated with body mass index in healthy adults. Neuropsychobiology 2006;53:153-156.

19. Neeper SA, Gomez-Pinilla F, Choi J, et al. Exercise and brain neurotrophins. Nature 1995;373:109.

20. Neeper SA, Gomez-Pinilla F, Choi J, et al. Physical activity increases mRNA for brain-derived neurotrophic factor and nerve growth factor in rat brain. Brain Res 1996; 726:49-56.

21. United States Census Bureau. FFF: Hispanic Heritage Month 2016. Accessed at: www.census.gov/newsroom/factsfor-features/2016/cb16-ff16.html on July 24, 2017.

22. Ogden CL, Carroll MD, Curtin LR, et al. Prevalence of overweight and obesity in the United States, 1999-2004. JAMA 2006;295:1549-1555.

23. Roberts CK, Hevener AL, Barnard RJ. Metabolic syndrome and insulin resistance: Underlying causes and modification by exercise training. Compr Physiol 2013;3:1-58.

24. Booth FW, Roberts CK, Laye MJ. Lack of exercise is a major cause of chronic diseases. Compr Physiol 2012;2: 1143-1211.

25. Shaibi GQ, Coletta DK, Vital V, et al. The design and conduct of a community-based registry and biorepository: A focus on cardiometabolic health in Latinos. Clin Transl Sci 2013;6:429-434.

26. Klein AB, Williamson R, Santini MA, et al. Blood BDNF concentrations reflect brain-tissue BDNF levels across species. Int J Neuropsychopharmacol 2011;14:347-353.

27. Hellweg R, von Arnim CA, Buchner M, et al. Neuroprotection and neuronal dysfunction upon repetitive inhibition of oxidative phosphorylation. Exp Neurol 2003;183:346354. 
28. DeMenna J, Puppala S, Chittoor G, et al. Association of common genetic variants with diabetes and metabolic syndrome related traits in the Arizona Insulin Resistance registry: A focus on Mexican American families in the Southwest. Hum Hered 2014;78:47-58.

29. Cotman CW, Berchtold NC. Exercise: A behavioral intervention to enhance brain health and plasticity. Trends Neurosci 2002;25:295-301.

30. Sleiman SF, Henry J, Al-Haddad R, et al. Exercise promotes the expression of brain derived neurotrophic factor (BDNF) through the action of the ketone body betahydroxybutyrate. Elife 2016;5. pii. e15092.

31. Gold SM, Schulz KH, Hartmann S, et al. Basal serum levels and reactivity of nerve growth factor and brain-derived neurotrophic factor to standardized acute exercise in multiple sclerosis and controls. J Neuroimmunol 2003;138:99-105.

32. Schmolesky MT, Webb DL, Hansen RA. The effects of aerobic exercise intensity and duration on levels of brainderived neurotrophic factor in healthy men. J Sports Sci Med 2013;12:502-511.

33. Szuhany KL, Bugatti M, Otto MW. A meta-analytic review of the effects of exercise on brain-derived neurotrophic factor. J Psychiatr Res 2015;60:56-64.

34. Shimizu E, Hashimoto K, Iyo M. Ethnic difference of the BDNF 196G/A (val66met) polymorphism frequencies: The possibility to explain ethnic mental traits. Am J Med Genet B Neuropsychiatr Genet 2004;126B:122-123.

35. Luykx JJ, Boks MP, Breetvelt EJ, et al. BDNF Val66Met homozygosity does not influence plasma BDNF levels in healthy human subjects. Prog Neuropsychopharmacol Biol Psychiatry 2013;43:185-187.

36. Wang Y, Zhang H, Li Y, et al. BDNF Val66Met polymorphism and plasma levels in Chinese Han population with obsessive-compulsive disorder and generalized anxiety disorder. J Affect Disord 2015;186:7-12.

37. Dogliotti G, Galliera E, Licastro F, et al. Age-related changes in plasma levels of BDNF in Down syndrome patients. Immun Ageing 2010;7:2.

Address correspondence to:

Yonas E. Geda, MD, MSc

Mayo Clinic Translational Neuroscience and Aging Program Mayo Clinic

13400 East Shea Boulevard Scottsdale, AZ 85259

E-mail: geda.yonas@mayo.edu 